\title{
Clinical outcomes for hilar and extrahepatic cholangiocarcinoma with adjuvant, definitive, or liver transplant-based neoadjuvant chemoradiotherapy strategies: a single-center experience
}

\author{
Brady S. Laughlin ${ }^{1}$, Molly M. Petersen ${ }^{2}$, Nathan Y. Yu ${ }^{1}$, Justin D. Anderson ${ }^{1}$, William G. Rule ${ }^{3}$, \\ Mitesh J. Borad ${ }^{4}$, Bashar A. Aqel ${ }^{5,6}$, Mohamad B. Sonbol ${ }^{4}$, Amit K. Mathur ${ }^{6,7}$, Adyr A. Moss ${ }^{6,7}$, \\ Tanios S. Bekaii-Saab ${ }^{4}$, Daniel H. Ahn ${ }^{4,8}$, Todd A. DeWees ${ }^{2,3}$, Terence T. Sio ${ }^{3}$, Jonathan B. Ashman ${ }^{3 \wedge}$ \\ ${ }^{1}$ Mayo Clinic School of Graduate Medical Education, Mayo Clinic College of Medicine and Science, Phoenix, Arizona, USA; ${ }^{2}$ Clinical Trials and \\ Biostatistics, Mayo Clinic, Scottsdale, Arizona, USA; ${ }^{3}$ Department of Radiation Oncology, Mayo Clinic Hospital, Phoenix, Arizona, USA; ${ }^{4}$ ivision \\ of Hematology and Medical Oncology, Mayo Clinic Hospital, Phoenix, Arizona, USA; ${ }^{5}$ Division of Gastroenterology and Hepatology, Mayo Clinic \\ Hospital, Phoenix, Arizona, USA; ${ }^{6}$ Transplant Center, Mayo Clinic Hospital, Phoenix, Arizona, USA; ${ }^{7}$ Division of Transplant and Hepatobiliary \\ Surgery, Mayo Clinic Hospital, Phoenix, Arizona, USA; ${ }^{8}$ Mayo Clinic Cancer Center, Mayo Clinic Hospital, Phoenix, Arizona, USA \\ Contributions: (I) Conception and design: TT Sio, JB Ashman; (II) Administrative support: TT Sio, JB Ashman; (III) Provision of study materials or \\ patients: TT Sio, JB Ashman; (IV) Collection and assembly of data: BS Laughlin; (V) Data analysis and interpretation: MM Petersen, TA DeWees; (VI) \\ Manuscript writing: All authors; (VII) Final approval of manuscript: All authors. \\ Correspondence to: Jonathan B. Ashman, MD, PhD. Department of Radiation Oncology, Mayo Clinic Hospital, 5777 E Mayo Blvd, Phoenix, AZ \\ 85054, USA. Email: Ashman.Jonathan@mayo.edu.
}

Background: We report our experience with 3 strategies for treating hilar and extrahepatic cholangiocarcinoma (CCA) including chemoradiotherapy: neoadjuvant chemoradiotherapy (nCRT) and orthotopic liver transplant, surgical resection and adjuvant chemoradiotherapy (aCRT), and definitive chemoradiotherapy (dCRT).

Methods: We included patients treated from 1998 through 2019. Kaplan-Meier estimates, log-rank testing, and univariate/multivariate Cox models were used to assess outcomes (local progression-free survival, disease-free survival, and overall survival).

Results: Sixty-five patients (nCRT, n=20; aCRT, n=16; dCRT, n=29) met inclusion criteria [median (range) age 65 years (27-84 years)]. Median posttreatment follow-up was 19.1 months (0.8-164.8 months) for all patients and 38.6, 24.3, and 9.0 months for the nCRT, aCRT, and dCRT groups, respectively. At 3 and 5 years, overall survival was $78 \%$ and $59 \%$ for the nCRT group; $47 \%$ and $35 \%$, aCRT group; and $11 \%$ and 0\%, dCRT group. Compared with the dCRT group, the nCRT group (hazard ratio $=0.13,95 \%$ CI: $0.05-0.33$ ) and the aCRT group (hazard ratio $=0.29,95 \%$ CI: $0.14-0.64$ ) had significantly improved overall survival $(\mathrm{P}<0.001)$. The 5 -year local progression-free survival $50 \%$ nCRT vs. 30\% aCRT vs. 0\% dCRT, $\mathrm{P}<0.001)$ and 5 -year disease-free survival (61\% nCRT vs. 30\% aCRT vs. $0 \%$ dCRT, $\mathrm{P}=0.01)$ were significantly better for strategies combined with surgery.

Conclusions: Outcomes for patients with extrahepatic CCA were superior for those who underwent nCRT/orthotopic liver transplant or postsurgical aCRT than for patients treated with dCRT. The excellent outcomes after nCRT/orthotopic liver transplant provide additional independent data supporting the validity of this strategy. The poor survival of patients treated with dCRT highlights a need for better therapies when surgery is not possible.

Keywords: Cholangiocarcinoma (CCA); hilar cholangiocarcinoma (hilar CCA); extrahepatic cholangiocarcinoma (extrahepatic CCA); liver transplant; neoadjuvant chemoradiation

^ ORCID: 0000-0001-7098-9465. 
Submitted Sep 21, 2021. Accepted for publication Jan 11, 2022

doi: 10.21037/jgo-21-615

View this article at: https://dx.doi.org/10.21037/jgo-21-615

\section{Introduction}

Cholangiocarcinoma (CCA) is an aggressive neoplasm of biliary epithelial origin. The location of these tumors in the biliary tree relative to the level of origin of the cystic duct is used to classify them as intrahepatic, hilar, or extrahepatic CCA. Hilar CCAs occur between the junction of the cystic duct and the secondary branches of the right and left hepatic ducts (1).

Various regimens including radiation therapy combined with concurrent chemotherapy have been used to treat CCA. In 1993, Mayo Clinic in Rochester, Minnesota, opened a clinical protocol for combining neoadjuvant external beam radiotherapy (EBRT) with concurrent 5 -fluorouracil and a brachytherapy boost followed by maintenance chemotherapy until orthotopic liver transplant (OLT) for patients with unresectable hilar or selected distal CCA (2). In the most recent report of patients in this protocol, overall survival (OS) at 5 years was $69 \%$ for those patients who successfully undergo transplantation (3). An alternative treatment option is surgical resection with negative margins and adjuvant chemoradiotherapy (aCRT), which is considered especially for patients with distal extrahepatic CCA $(4,5)$. However, even with surgical resection, the 5 -year OS may only be $20 \%$ to $40 \%(5-7)$. If neither a transplant nor surgical resection is possible, chemotherapy and EBRT can be used with curative intent. Unfortunately, the prognosis for patients whose tumors are managed by definitive chemoradiotherapy (dCRT) remains poor, with reported median OS of 10 to 24 months and 3 -year OS of $10 \%$ to $20 \%$ (8-13).

The purpose of this investigation was to evaluate outcomes of patients with hilar and distal CCA in which chemoradiation was included as part of the treatment strategy. Studies reported in the literature have shown that patients with CCA undergoing transplant have excellent outcomes compared with those undergoing only surgical resection and/or radiotherapy. We hypothesized that patients with hilar or distal CCA who undergo neoadjuvant chemoradiotherapy (nCRT) followed by OLT will have better outcomes than patients who have radiotherapy and surgical resection. Herein, we report results for patients with distal and hilar CCA who were treated at our tertiary care cancer center with either nCRT followed by OLT, surgical resection followed by aCRT, or dCRT. We present the following article in accordance with the STROBE reporting checklist (available at https://jgo.amegroups.com/ article/view/10.21037/jgo-21-615/rc).

\section{Methods}

The study was approved by the Mayo Clinic Institutional Review Board (No. 19-007493) and was conducted in accordance with the Declaration of Helsinki (as revised in 2013). Because of the retrospective nature of the study, the requirement for informed consent was waived. We retrospectively reviewed electronic health records to identify patients with hilar or distal CCA treated from June 26, 1998, through September 20, 2019. Patients who had the following treatment regimens were included: nCRT and maintenance chemotherapy followed by OLT, surgical resection followed by aCRT, or dCRT.

Patients were selected for each treatment based on anatomic factors, staging, and medical comorbid conditions. Patients were always considered first for transplant or surgery. Patients with hilar CCA were screened for eligibility for nCRT and OLT as the preferred treatment. Patients with distal CCA were evaluated for primary surgery. Eligibility for patients undergoing nCRT and OLT was defined per the Mayo Clinic protocol $(2,14)$. Patients in this group had CCA deemed unresectable because of anatomy in de novo cases or because of underlying primary sclerosing cholangitis. Diagnosis was made by a positive intraluminal biopsy, brush cytology, biliary aneuploidy identified on fluorescence in situ hybridization analysis, or cancer antigen (CA) $19-9$ more than $100 \mathrm{U} / \mathrm{mL}$ in the presence of a malignant-appearing stricture. Patients were excluded if they had metastatic disease, tumor size greater than $3 \mathrm{~cm}$ on preoperative imaging, a nonendoscopic biopsy, or a medical condition precluding transplant. Radiation planning and delivery techniques included photon intensitymodulated radiotherapy (IMRT), 3-dimensional conformal radiotherapy, or proton beam therapy. Chemotherapy concurrent with EBRT was prescribed with either continuous infusion 5 -fluorouracil or capecitabine. After chemoradiotherapy, capecitabine was given as maintenance therapy until OLT. Before OLT, an exploratory surgical 
procedure was performed to evaluate for extrahepatic disease, which included biopsies of lymph nodes along the common bile duct and proper hepatic artery as well as biopsies of any other lymph nodes or intraabdominal findings suggestive of cancer. In the aCRT group, radical resection for hilar or distal CCA was performed, which included resection of the extrahepatic biliary tree with or without hepatic resection (partial or total) or a Whipple procedure, depending on the anatomic extension of disease in the biliary tree. Patients who were not eligible for OLT or radical surgery for anatomic or medical reasons but did not have distant metastatic disease on staging received dCRT.

\section{Statistical analysis}

We compared differences in patient, tumor, and treatment characteristics among the 3 groups and analyzed the results using the Kruskal-Wallis test for continuous variables and the Fisher exact test for categorical variables. Time-to-event analyses were calculated from the date of transplant in the nCRT group or from the end of radiotherapy for the aCRT and dCRT groups. The primary end point was OS, which was defined as the time from completion of treatment until the last follow-up date or death. Secondary end points were local progression-free survival (LPFS), defined as the time from treatment completion to local disease progression, and disease-free survival (DFS), defined as the time from treatment completion to either local or distant disease progression. Kaplan-Meier estimates were used to determine the risk of event as a function of time (15). Univariate and multivariate Cox models were used to determine variables associated with OS, LPFS, and DFS. Adverse events were graded according to the Common Terminology Criteria of Adverse Events version 5.0. Kaplan-Meier estimates, hazard ratios (HRs), and ORs were calculated along with the 95\% CI. Analyses were performed using R version 3.6.2 (R Foundation for Statistical Computing) and SAS version 9.3 (SAS Institute Inc). P values less than 0.05 were considered statistically significant.

\section{Results}

\section{Patient and tumor characteristics}

As of September 20, 2019, 65 patients with hilar or distal CCA received radiotherapy as part of curative intent treatment and were included in this analysis. Of those
65 patients, 20 patients were treated with nCRT and OLT, 16 patients underwent surgical resection with aCRT, and 29 patients were treated with dCRT. Table 1 documents patient and tumor characteristics. The median age at diagnosis was 65 years for all patients and 59, 68, and 72 years for the nCRT, aCRT, and dCRT cohorts, respectively. The median baseline CA 19-9 was $102 \mathrm{U} / \mathrm{mL}$ for all patients and 43.0, 55.7, and 294.5 for the nCRT, aCRT, and dCRT cohorts, respectively. Of the patients, 17 (26\%) had ulcerative colitis, and 19 (29\%) had primary sclerosing cholangitis.

\section{Treatment characteristics}

The treatment characteristics for each patient cohort are summarized in Table 2. Of the patients undergoing radical resection in the aCRT group, 4 patients with perihilar disease underwent bile duct resection, partial hepatectomy, and Roux-en-Y hepaticojejunostomy and 12 patients with distal bile duct disease underwent a Whipple procedure (pancreaticoduodenectomy, cholecystectomy, and common bile duct resection). All patients in the nCRT group had treatment to an initial field, which included the primary and adjacent draining nodal basin lymph nodes, with 45 Gy in 30 fractions with twice daily fractionations of 1.5 Gy. A boost to the primary tumor was then delivered by additional EBRT (6-15 Gy, 1.5 Gy twice daily). For the patients receiving dCRT and aCRT, radiotherapy was delivered with a once-daily fractionation. The boost dose delivered varied between 5.4 to 20 Gy. The total EBRT dose was higher for patients in the nCRT group than for patients in the aCRT and dCRT groups (54.0 vs. 50.4 vs. 50.4 Gy; $\mathrm{P}<0.01)$. An intraluminal brachytherapy boost of low-doserate iridium-192 was delivered via an endoscopically guided catheter to 3 patients who received dCRT and 2 who received nCRT.

\section{Survival analysis}

The median (range) follow-up from the end of treatment was $19.1(0.8-164.8)$ months, with median follow-up for the nCRT group of 38.6 months; aCRT, 24.3 months; and dCRT, 9.0 months. By the end of follow-up, 40 patients had died: $6(30 \%)$ in the nCRT group, $10(63 \%)$ in the aCRT group, and $24(83 \%)$ in the dCRT group. Local progression occurred in 9 patients by the end of follow-up: $0(0 \%)$ in the nCRT group, $4(25 \%)$ in the aCRT group, 
Table 1 Patient characteristics

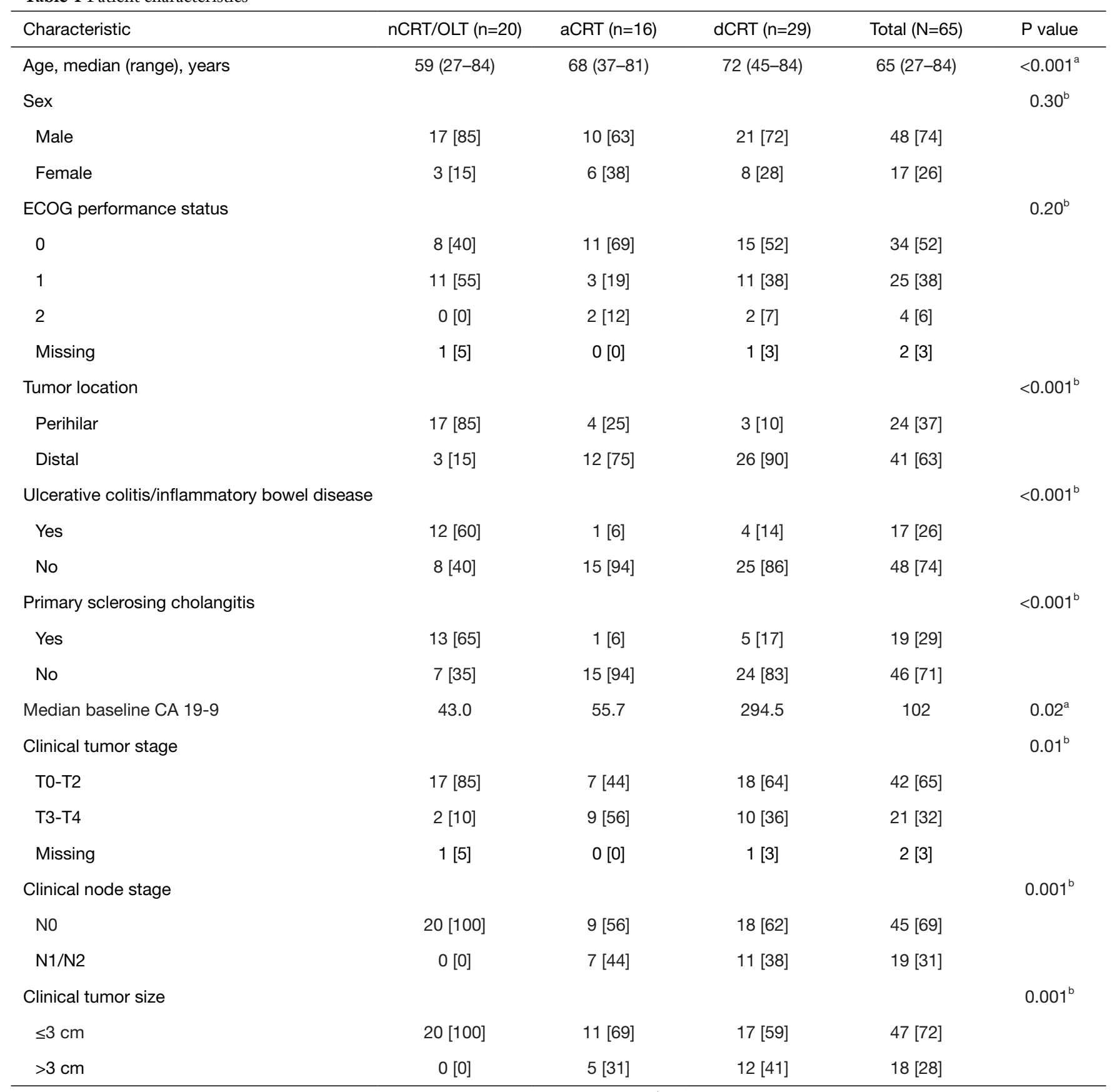

Data are presented as No. [\%] unless otherwise indicated. ${ }^{a}$, Kruskal-Wallis test; ${ }^{b}$, Fisher exact test. aCRT, adjuvant chemoradiotherapy; dCRT, definitive chemoradiotherapy; ECOG, Eastern Cooperative Oncology Group; nCRT/OLT, neoadjuvant chemoradiotherapy followed by orthotopic liver transplantation.

and $5(17 \%)$ in the dCRT group. By the end of followup, disease progression occurred in 4 patients $(20 \%)$ in the nCRT group, 8 patients (50\%) in the aCRT group, and 11 patients $(38 \%)$ in the dCRT group. Compared with the dCRT group, the nCRT group (HR $=0.13,95 \% \mathrm{CI}$ : 0.05-0.33) and aCRT group (HR $=0.29,95 \%$ CI: 0.14-0.64) had significantly improved OS (Figure $1 ; \mathrm{P}<0.001$ ). At 3 and 5 years, OS was $78 \%$ and $59 \%$ for the nCRT group, $49 \%$ 
Table 2 Treatment characteristics

\begin{tabular}{|c|c|c|c|c|c|}
\hline Characteristics & $\mathrm{nCRT} / \mathrm{OLT}(\mathrm{n}=20)$ & Surgical resection/aCRT $(n=16)$ & dCRT $(n=29)$ & Total $(\mathrm{N}=65)$ & $P$ value \\
\hline 3D CRT & 5 [25] & $11[69]$ & $16[57]$ & 32 [49] & $0.009^{b}$ \\
\hline IMRT & $15[75]$ & $4[25]$ & $13[43]$ & 32 [49] & \\
\hline Proton & $0[0.0]$ & $1[6]$ & $0[0.0]$ & $1[2]$ & \\
\hline Mean total RT dose, Gy [range] & $54.0[45.0-60.0]$ & $50.4[50.0-59.4]$ & $50.4[7.2-62.4]$ & $54.0[7.2-62.4]$ & $0.005^{\mathrm{a}}$ \\
\hline Fractions & 30 [23-30] & 25 [25-30] & 25 [4-30] & $27[4-30]$ & $<0.001^{\mathrm{a}}$ \\
\hline \multicolumn{6}{|l|}{ Fractionation schedule } \\
\hline Once daily & $1[5]$ & $16[100]$ & 29 [100] & $46[71]$ & $<0.001^{\text {b }}$ \\
\hline$\leq 59.5$ Gy & $2[10]$ & $9[56]$ & $14[48]$ & 25 [38] & $0.004^{b}$ \\
\hline$>59.5$ Gy & $18[90]$ & $7[44]$ & 15 [52] & 40 [62] & \\
\hline \multicolumn{6}{|l|}{ Brachytherapy boost } \\
\hline Yes & $3[15]$ & $0[0]$ & $3[10]$ & 6 [9] & $0.36^{\mathrm{b}}$ \\
\hline No & 17 [85] & 16 [100] & 26 [90] & 59 [91] & \\
\hline Brachytherapy boost dose, Gy & $20.0[10.0-20.0]$ & 0 & $10.0[8.0-20.0]$ & $15.0[8.0-20.0]$ & $0.35^{a}$ \\
\hline Total boost dose, Gy [range] & $9.0[0-15.0]$ & $5.4[0-14.4]$ & $0[0-13.0]$ & $5.4[0-15.0]$ & $<0.001^{2}$ \\
\hline Total boost fractions & $6[0-10]$ & $3[0-8]$ & $0[0-6]$ & $3[0-10]$ & $<0.001^{2}$ \\
\hline
\end{tabular}

Data are presented as No. [\%] unless otherwise indicated. ${ }^{a}$, Kruskal-Wallis test; ${ }^{b}$, Fisher exact test. aCRT, adjuvant chemoradiotherapy; BED, biologically effective dose; dCRT, definitive chemoradiotherapy; 3D CRT, 3-dimensional conformal radiotherapy; nCRT/OLT, neoadjuvant chemoradiotherapy followed by orthotopic liver transplantation; IMRT, intensity-modulated radiotherapy; RT, radiotherapy.

and $38 \%$ for the aCRT group, and $16 \%$ and $0 \%$ for the dCRT group. For the 3 groups, the 5 -year LPFS was $50 \%$, nCRT; 30\%, aCRT; and 0\%, dCRT (Figure 2; P<0.001). At 5 years, DFS was $61 \%$ in the nCRT group versus $30 \%$ in the aCRT group and $0 \%$ in the dCRT group.

Univariate analysis with clinical outcome association showed that treatment strategy, negative lymph node status, smaller tumor size, younger patient age, and low baseline CA 19-9 were significantly associated with improved clinical outcomes (all $\mathrm{P}<0.05$ ) (Table 3). In multivariate analysis, treatment strategy for the $\mathrm{nCRT}$ group remained the only variable related to improved $\mathrm{OS}(\mathrm{P}=0.02)$.

\section{Adverse events}

Two patients in the dCRT group died after their radiotherapy was interrupted at 7.2 and 13.5 Gy. One patient had intractable nausea and vomiting and a subsequent finding of cholangitis. Given the patient's deteriorating condition, dCRT was stopped and palliative care was provided. The other patient developed rhabdomyolysis that was attributed to capecitabine and atorvastatin, and this patient was unable to continue treatment. Of the patients, 20 were hospitalized during chemoradiotherapy treatment: 6 patients $(30 \%)$ in the nCRT group, 2 patients $(13 \%)$ in 


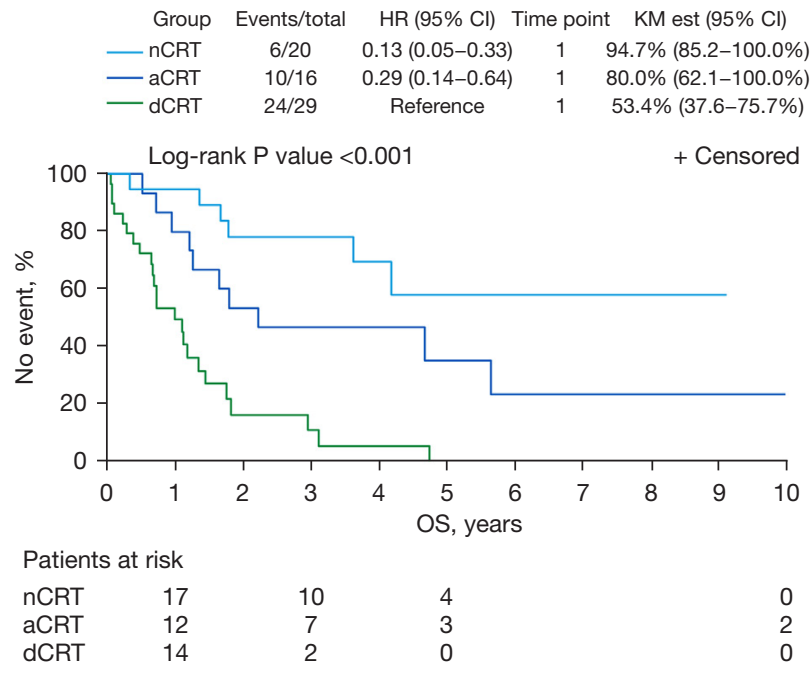

Figure 1 OS for patients who underwent nCRT followed by OLT, aCRT after radical resection, or dCRT. HR, hazard ratio; KM est, Kaplan-Meier estimate; OS, overall survival; nCRT, neoadjuvant chemoradiotherapy; OLT, orthotopic liver transplant; aCRT, adjuvant chemoradiotherapy; dCRT, definitive chemoradiotherapy.

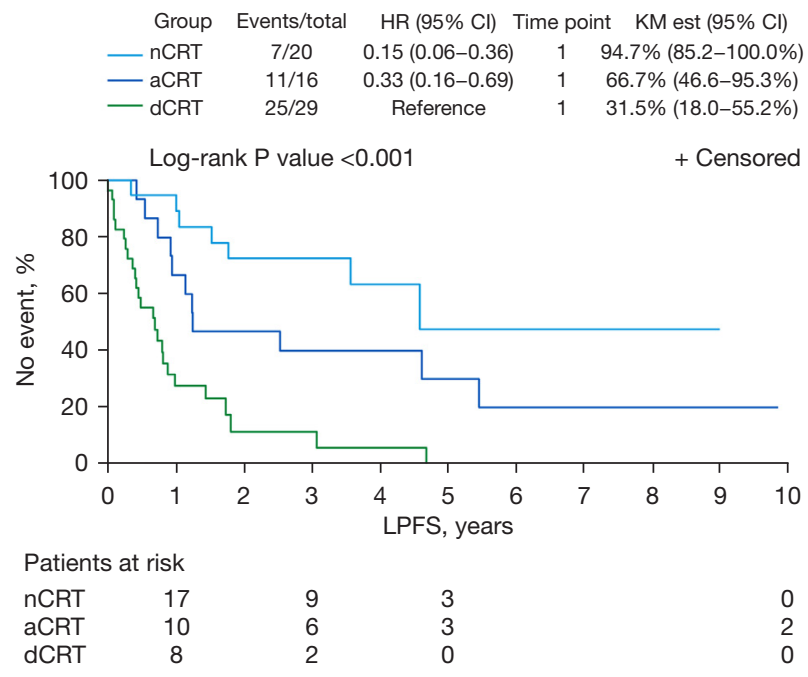

Figure 2 LPFS for patients undergoing nCRT followed by liver transplant, aCRT after radical resection, or dCRT. HR, hazard ratio; KM est, Kaplan-Meier estimate; LPFS, local progressionfree survival; nCRT, neoadjuvant chemoradiotherapy; aCRT, adjuvant chemoradiotherapy; dCRT, definitive chemoradiotherapy.

the aCRT group, and 12 patients (41\%) in the dCRT group. Treatment breaks occurred for 22 patients overall (34\%); 6 (30\%) during nCRT, 2 (13\%) during aCRT, and 14 (48\%) during dCRT. Biliary infection was the most common adverse event, occurring in 11 patients $(55 \%)$ treated with nCRT, 5 (31\%) treated with aCRT, and 17 (59\%) treated with dCRT. Three patients had grade 3 late gastrointestinal toxicity. Duodenal ulcers were attributed to radiotherapy in 1 patient who had aCRT and 2 patients who had dCRT. No patients developed gastrointestinal luminal perforation or fistula. No differences were identified in acute or late gastrointestinal toxicities among the different groups.

\section{Discussion}

We reported herein our single-institution experience using chemoradiotherapy as a component of various treatment strategies in the multidisciplinary care of patients with hilar and extrahepatic CCA. Given that CCA is a relatively rare malignancy, our series offers a large experience of the most typical curative strategies from a tertiary care cancer center. In our series, the 5 -year OS for patients receiving nCRT and OLT was $59 \%$. This result is consistent with the updated results from Mayo Clinic that showed OS at 5 years of $51 \%$ for all patients at the start of nCRT and $69 \%$ for patients after OLT (3). Therefore, our study adds results to the literature for an independent cohort of patients that further validates the approach of nCRT followed by OLT in appropriately selected patients.

Our outcomes are also consistent with previously reported experience. The best outcomes for patients with CCA needing multimodality therapy have been achieved with a combination of nCRT followed by OLT. However, outcomes have been reported only from a small number of centers using this approach. With liver transplant alone, reported outcomes were poor. In a report from the University of Cincinnati that included 207 patients, the 5 -year survival rate was $23 \%$ with transplant alone (16). In addition, $51 \%$ of their patients had recurrence after transplant, the majority (84\%) occurring within 2 years. Robles et al. (17) reported a Spanish experience with similar results: a 5 -year OS rate of $30 \%$ for patients undergoing transplant alone for hilar CCA. The main cause of patient death was recurrence of CCA, which occurred in $53 \%$ of patients. The poor outcomes for liver transplant alone led clinicians at Mayo Clinic in Rochester, Minnesota, to develop their protocol for nCRT before OLT (2). Patients in the original series who underwent transplant had an $82 \%$ OS rate at 5 years, which demonstrated the promise of the regimen $(18,19)$. Several studies have provided updated outcomes with increased patient numbers and 
Table 3 Univariate associations with OS and DFS

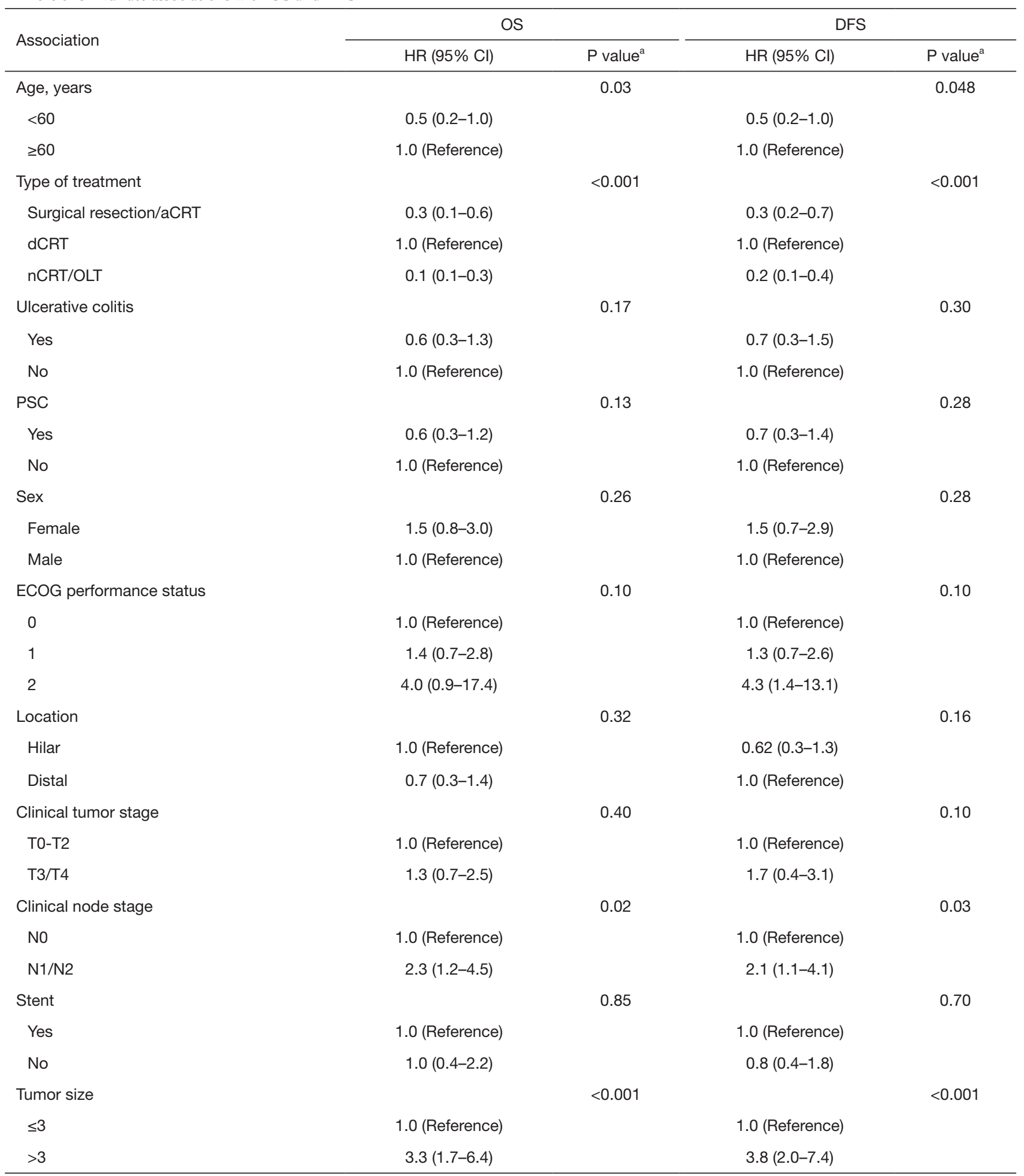

a , Type 3 likelihood-ratio P value. aCRT, adjuvant chemoradiotherapy; dCRT, definitive chemoradiotherapy; DFS, disease-free survival; ECOG, Eastern Cooperative Oncology Group; nCRT/OLT, neoadjuvant chemoradiotherapy followed by orthotopic liver transplantation; OS, overall survival; PSC, primary sclerosing cholangitis. 
longer follow-up $(3,14,20)$. However, the excellent results from the previous Mayo Clinic study have had limited corroboration outside of this single, high-volume center (2). A retrospective study from 12 institutions reported 53\% OS at 5 years (21). Although no statistically significant difference in OS between patients treated at Mayo Clinic and the other 11 centers was identified, $67 \%$ of all patients were treated at Mayo Clinic.

Our results also question the necessity of brachytherapy in the pretransplant neoadjuvant protocol. Following the initial course of EBRT of 45 Gy twice daily, 18 of 20 patients $(90 \%)$ in the nCRT group received a boost with additional EBRT; only 2 patients had a boost with brachytherapy. In the multi-institutional series, $25 \%$ of patients did not receive brachytherapy, and no difference in recurrence-free survival was identified when patients were compared with those treated with a brachytherapy boost (22).

Although patients who had nCRT combined with OLT had better survival outcomes, concern remained that cancer would recur in these patients. In 2006, Mayo Clinic updated their series to identify prognostic factors (18). That study reported disease recurrence for 11 of 65 patients $(17 \%)$ treated according to the protocol. The risk factors for recurrence identified from the study were patient age, CA 19-9 greater than $100 \mathrm{U} / \mathrm{mL}$ before transplant, prior cholecystectomy, discreet mass on transplant imaging, residual tumor greater than $2 \mathrm{~cm}$ in the explant specimen, tumor grade, and perineural invasion. These findings led to revision of eligibility criteria. In our current study, negative lymph node status, smaller tumor size, younger patient age, and low baseline CA 19-9 were significantly associated with improved clinical outcomes.

The current study also showed that nCRT and OLT provide superior outcomes to surgical resection with aCRT (5-year OS, $59 \%$ vs. $39 \%$; $\mathrm{P}<0.001$ ). It is acknowledged that patients eligible for nCRT/OLT are a highly selected group with potentially more favorable risk factors than patients treated with surgery and aCRT. However, treatment strategy was identified as an independent prognostic factor on multivariate analysis. This finding is also consistent with prior data from a Mayo Clinic report (20) that showed a 5 -year OS after radical resection of $21 \%$, but aCRT was used only in selected patients. A series of 373 patients undergoing surgical resection without transplant had 1-year, 3 -year, and 5 -year survival rates of $32.5 \%, 9.2 \%$, and $4.1 \%$, respectively (23), and aCRT was associated with OS. Our results for the group of patients treated with aCRT after radical resection compared favorably with these previous outcomes.

Patients with hilar or distal CCA whose anatomy or comorbid conditions prohibit surgical interventions have worse outcomes than patients treated with surgery. In our current series, patients who received dCRT had 16\% OS at 3 years, and no patients survived at 5 years. Poor outcomes can be attributed to multiple factors, including the high risk of both local and distant relapse, morbidity associated with treatment, and prior medical comorbid conditions. Multiple single-institution series have demonstrated similar outcomes for patients treated solely with dCRT, with 3 -year OS rates ranging from $10 \%$ to $20 \%$ and median survival between 10 and 24 months (8-12). Bisello et al. (24) reported a median survival of 10 months for 76 patients treated between 1991 and 2017 with doses of 30 to 85 Gy in addition to concurrent 5-fluorouracil. In a large series of 209 patients, Yoshioka et al. (13) reported 2-year OS of $30 \%$. It would be reasonable to conclude that dCRT with traditional radiotherapy dose techniques and fluorouracilbased chemosensitization is not truly curative but instead only palliative. However, studies have demonstrated more promising outcomes when radiation dose escalation can be achieved safely (25).

Adverse events associated with progressive disease and radiotherapy effect can be quite profound. Approximately $20 \%$ to $30 \%$ of patients experience grade 3 toxicity, and late complications such as bleeding or intestinal ulceration can occur in $10 \%$ to $20 \%$ of patients $(8-13,26)$. Acute cholangitis was a common complication in this series of patients undergoing CRT, although only 3 patients (4.6\%) had late grade 3 gastrointestinal toxicity.

A major limitation of this study is its retrospective design and inherent bias in patient selection for each treatment pathway. Because of strict eligibility criteria, patients undergoing the nCRT with OLT were younger, had less advanced disease as indicated by a higher proportion of lower $\mathrm{T}$ stages, and had more favorable risk factors such as primary sclerosing cholangitis. Other limitations included the small patient population included in this study. Finally, this retrospective analysis included patients treated over a 20-year period during which multiple advances occurred in diagnostic procedures, surgical techniques, radiotherapy planning, and adjuvant therapies.

Despite these limitations, the results of this study are important because they reaffirm that cure can be achieved for patients with hilar or extrahepatic CCA who undergo nCRT and OLT or surgical resection with aCRT. Further efforts to refine the nCRT protocol are warranted, given the 
improvements in EBRT techniques such as IMRT, imageguided radiotherapy, and proton beam therapy. For patients undergoing dCRT, these technical advances in radiotherapy should be combined with novel molecular diagnostic methods and advanced systemic therapies to improve the poor outcomes of patients who are ineligible for OLT or radical surgical resection.

\section{Conclusions}

For patients with extrahepatic CCA, outcomes were superior for those who underwent nCRT followed by OLT or aCRT after radical resection than for patients treated only with dCRT. Given the retrospective study design, these outcomes were influenced by patient selection bias. Nevertheless, our data support a strategy wherein patients are first evaluated for the transplant protocol or surgical resection whenever possible. Our experience using nCRT and OLT is 1 of only a few studies to independently reproduce similar results from those of the earlier Mayo Clinic study. The delivery of dCRT needs to be improved through technical advances or novel combined modality therapies.

\section{Acknowledgments}

Data were presented in part at the American Society of Clinical Oncology Gastrointestinal Cancers Symposium, San Francisco, California, January 23-25, 2020, and the American Society for Radiation Oncology 62nd Annual Meeting, Virtual, October 24-28, 2020.

Funding: None.

\section{Footnote}

Reporting Checklist: The authors have completed the STROBE reporting checklist. Available at https://jgo. amegroups.com/article/view/10.21037/jgo-21-615/rc

Data Sharing Statement: Available at https://jgo.amegroups. com/article/view/10.21037/jgo-21-615/dss

Peer Review File: Available at https://jgo.amegroups.com/ article/view/10.21037/jgo-21-615/prf

Conflicts of Interest: All authors have completed the ICMJE uniform disclosure form (available at https://jgo.amegroups. com/article/view/10.21037/jgo-21-615/coif). DHA is an advisor for Genentech, Eisai, Advanced Accelerator Applications, Exelixis, and Daiichi Sankyo. TTS provides strategic and scientific recommendations as a member of the Advisory Board and speaker for Novocure, Inc., which is not in any way associated with the content presented in this manuscript. TSBS receives research funding from Agios, Arys, Arcus, Atreca, Boston Biomedical, Bayer, Amgen, Merck, Celgene, Lilly, Ipsen, Clovis, Seattle Genetics, Genentech, Novartis, Mirati, Merus, Abgenomics, Incyte, Pfizer, and BMS; has consulting relationships with Ipsen, Arcus, Array Biopharma, Pfizer, Seattle Genetics, Bayer, Genentech, Incyte, Merck, Stemline, AbbVie, Boehringer Ingelheim, Janssen, Eisai, Daichii Sankyo, Natera, TreosBio, Celularity, Exact Science, Sobi, Beigene, Kanaph, Xilis, Astra Zeneca, and Foundation Medicine; participates on the Data Safety Monitoring Boards of Fibrogen, Suzhou Kintor, Astra Zeneca, Exelixis, Lilly, PanCan, and 1Globe; is a member of the Scientific Advisory Boards of Imugene, Immuneering, and Panbela Therapeutics; and has the following patents pending: WO/2018/183488 and WO/2019/055687. The other authors have no conflicts of interest to declare.

Ethical Statement: The authors are accountable for all aspects of the work and in ensuring that questions related to the accuracy or integrity of any part of the work are appropriately investigated and resolved. The study was approved by the Mayo Clinic Institutional Review Board (No. 19-007493) and was conducted in accordance with the Declaration of Helsinki (as revised in 2013). Because of the retrospective nature of the study, the requirement for informed consent was waived.

Open Access Statement: This is an Open Access article distributed in accordance with the Creative Commons Attribution-NonCommercial-NoDerivs 4.0 International License (CC BY-NC-ND 4.0), which permits the noncommercial replication and distribution of the article with the strict proviso that no changes or edits are made and the original work is properly cited (including links to both the formal publication through the relevant DOI and the license). See: https://creativecommons.org/licenses/by-nc-nd/4.0/.

\section{References}

1. Gores GJ. Cholangiocarcinoma: current concepts and insights. Hepatology 2003;37:961-9.

2. De Vreede I, Steers JL, Burch PA, et al. Prolonged disease- 
free survival after orthotopic liver transplantation plus adjuvant chemoirradiation for cholangiocarcinoma. Liver Transpl 2000;6:309-16.

3. Tan EK, Rosen CB, Heimbach JK, et al. Living Donor Liver Transplantation for Perihilar Cholangiocarcinoma: Outcomes and Complications. J Am Coll Surg 2020;231:98-110.

4. Horgan AM, Amir E, Walter T, et al. Adjuvant therapy in the treatment of biliary tract cancer: a systematic review and meta-analysis. J Clin Oncol 2012;30:1934-40.

5. Rea DJ, Munoz-Juarez M, Farnell MB, et al. Major hepatic resection for hilar cholangiocarcinoma: analysis of 46 patients. Arch Surg 2004;139:514-23; discussion 523-5.

6. Washburn WK, Lewis WD, Jenkins RL. Aggressive surgical resection for cholangiocarcinoma. Arch Surg 1995;130:270-6.

7. Jarnagin WR, Fong Y, DeMatteo RP, et al. Staging, resectability, and outcome in 225 patients with hilar cholangiocarcinoma. Ann Surg 2001;234:507-17; discussion 517-9.

8. Deodato F, Clemente G, Mattiucci GC, et al. Chemoradiation and brachytherapy in biliary tract carcinoma: long-term results. IntJ Radiat Oncol Biol Phys 2006;64:483-8.

9. Brunner TB, Schwab D, Meyer T, et al. Chemoradiation may prolong survival of patients with non-bulky unresectable extrahepatic biliary carcinoma. A retrospective analysis. Strahlenther Onkol 2004;180:751-7.

10. Torgeson A, Lloyd S, Boothe D, et al. Chemoradiation Therapy for Unresected Extrahepatic Cholangiocarcinoma: A Propensity Score-Matched Analysis. Ann Surg Oncol 2017;24:4001-8.

11. Ben-David MA, Griffith KA, Abu-Isa E, et al. External-beam radiotherapy for localized extrahepatic cholangiocarcinoma. Int J Radiat Oncol Biol Phys 2006;66:772-9.

12. Elganainy D, Holliday EB, Taniguchi CM, et al. Dose escalation of radiotherapy in unresectable extrahepatic cholangiocarcinoma. Cancer Med 2018;7:4880-92.

13. Yoshioka Y, Ogawa K, Oikawa H, et al. Impact of intraluminal brachytherapy on survival outcome for radiation therapy for unresectable biliary tract cancer: a propensity-score matched-pair analysis. Int J Radiat Oncol Biol Phys 2014;89:822-9.

14. Heimbach JK, Gores GJ, Haddock MG, et al. Predictors of disease recurrence following neoadjuvant chemoradiotherapy and liver transplantation for unresectable perihilar cholangiocarcinoma. Transplantation 2006;82:1703-7.

15. Kaplan EL, Meier P. Nonparametric Estimation from Incomplete Observations. J Am Stat Assoc 1958;53:457-81.
16. Meyer CG, Penn I, James L. Liver transplantation for cholangiocarcinoma: results in 207 patients. Transplantation 2000;69:1633-7.

17. Robles R, Figueras J, Turrión VS, et al. Spanish experience in liver transplantation for hilar and peripheral cholangiocarcinoma. Ann Surg 2004;239:265-71.

18. Rosen CB, Heimbach JK, Gores GJ. Liver transplantation for cholangiocarcinoma. Transpl Int 2010;23:692-7.

19. Heimbach JK, Gores GJ, Haddock MG, et al. Liver transplantation for unresectable perihilar cholangiocarcinoma. Semin Liver Dis 2004;24:201-7.

20. Rea DJ, Heimbach JK, Rosen CB, et al. Liver transplantation with neoadjuvant chemoradiation is more effective than resection for hilar cholangiocarcinoma. Ann Surg 2005;242:451-8; discussion 458-61.

21. Darwish Murad S, Kim WR, Harnois DM, et al. Efficacy of neoadjuvant chemoradiation, followed by liver transplantation, for perihilar cholangiocarcinoma at 12 US centers. Gastroenterology 2012;143:88-98.e3; quiz e14.

22. Darwish Murad S, Kim WR, Therneau T, et al. Predictors of pretransplant dropout and posttransplant recurrence in patients with perihilar cholangiocarcinoma. Hepatology 2012;56:972-81.

23. Jan YY, Yeh CN, Yeh TS, et al. Prognostic analysis of surgical treatment of peripheral cholangiocarcinoma: two decades of experience at Chang Gung Memorial Hospital. World J Gastroenterol 2005;11:1779-84.

24. Bisello S, Buwenge M, Palloni A, et al. Radiotherapy or Chemoradiation in Unresectable Biliary Cancer: A Retrospective Study. Anticancer Res 2019;39:3095-100.

25. Jethwa KR, Sannapaneni S, Mullikin TC, et al. Chemoradiotherapy for patients with locally advanced or unresectable extra-hepatic biliary cancer. J Gastrointest Oncol 2020;11:1408-20.

26. Foo ML, Gunderson LL, Bender CE, et al. External radiation therapy and transcatheter iridium in the treatment of extrahepatic bile duct carcinoma. Int J Radiat Oncol Biol Phys 1997;39:929-35.

Cite this article as: Laughlin BS, Petersen MM, Yu NY, Anderson JD, Rule WG, Borad MJ, Aqel BA, Sonbol MB, Mathur AK, Moss AA, Bekaii-Saab TS, Ahn DH, DeWees TA, Sio TT, Ashman JB. Clinical outcomes for hilar and extrahepatic cholangiocarcinoma with adjuvant, definitive, or liver transplant-based neoadjuvant chemoradiotherapy strategies: a single-center experience. J Gastrointest Oncol 2022;13(1):288-297. doi: 10.21037/jgo-21-615 